\title{
Força muscular do assoalho pélvico entre mulheres continentes jovens e climatéricas
}

\author{
Muscle strength of the pelvic floor among young and \\ climateric continent women
}

\author{
Eliane Cristina Hilberath Moreira ${ }^{1 *}$; Paula Bueno de Arruda $^{2}$
}

\begin{abstract}
Resumo
O climatério representa a fase em que ocorrem modificações no trato uroginecológico, como a diminuição da força muscular do assoalho pélvico e turgidez uretral. É sugerido que músculos sadios são volumosos e isso os capacita a suportar as paredes da vagina tão bem como prover uma ação esfincteriana, favorecendo o fechamento e a continência uretral. É plausível que o suprimento de sangue associado a músculos fortes promoverá a saúde do epitélio na área, estimulando a lubrificação vaginal adequada e o retardo das mudanças atróficas da idade. O objetivo deste estudo foi verificar a força muscular do assoalho pélvico entre mulheres saudáveis jovens e climatéricas, buscando identificar as alterações existentes nesta musculatura. O estudo foi composto por 49 mulheres dividas em dois grupos: Grupo 1 composto por mulheres ( $\mathrm{N}=24)$ com média de idade de 29,33 anos e o grupo 2 composto por mulheres $(\mathrm{N}=25)$ com média de idade de 51,8 anos, submetidas a avaliação da força muscular do assoalho pélvico por meio de palpação bidigital e perineometria. O presente estudo não identificou diferenças significativas da força de pressão muscular do assoalho pélvico pela perineometria entre os grupos estudados. Na avaliação pela palpação bidigital, notou-se uma capacidade maior de contração voluntária e sustentação desta contração nas mulheres com faixa etária entre 25 a 35 anos.

Palavras-chave: Força muscular. Assoalho pélvico. Perineometria.
\end{abstract}

\begin{abstract}
The menopause represents a stage in life when modifications in the urogynecological tract occur as a decrease in the muscle strength of the pelvic floor and urethral turgidity. It is suggested that healthy muscles are thick and this enables them to support the vagina walls as well as provide a sphincteral action, favoring the closing and urethral continence. It is possible that the blood supply associated to strong muscles will promote the health of the epithelium in the area, stimulating the adequate vaginal lubrication and the delay of atrophic changes due to the age. The purpose of this study was to verify the muscle strength of the pelvic floor among healthy young and climateric women, in order to identify the existing alterations in this musculature. The study consisted of 49 women divided in two groups: Group 1 consisting of women $(\mathrm{N}=24)$ with an average age of 29.33 years old and Group 2, consisting of women $(\mathrm{N}=25)$ with an average age of 51.8 years old, submitted to the evaluation of the muscle strength of the pelvic floor by means of bidigital palpation and perineometry. Significant differences were not identified concerning the pelvic floor muscle strength by means of the perineometry in the studied groups. As for the bidigital palpation evaluation, a larger capacity of voluntary contraction and sustained maintenance of this contraction in 25 to 35 -years old women was observed.
\end{abstract}

Key-words: Muscle strength. Pelvic floor. Perineometry.

\footnotetext{
${ }^{1}$ Docente Adjunto do Departamento de Fisioterapia da Universidade Estadual de Londrina; mestre e doutora em Ginecologia e Obstetrícia-FMB-UNESP-Botucatu.E-mail: liamoreira@uel.br; liamoreira@sercomtel.com.br

* Autor (a) para correspondência

${ }^{2}$ Aluna do $4^{\circ}$ ano do curso de Fisioterapia da Universidade Estadual de Londrina - bolsista PIBIC/CNPQ
} 


\section{Introdução}

Segundo o grupo de avaliação clínica do assoalho pélvico da Sociedade Internacional de Continência, o termo "músculos do assoalho pélvico" (MAP) refere a camada muscular que auxilia no suporte dos órgãos pélvicos. Esses músculos são os responsáveis pelo fechamento do hiato urogenital, quando se contraem, e desempenham importante papel na manutenção da continência de urina e de conteúdo retal (MESSELINK et al., 2005).

A importância do papel dos músculos do assoalho pélvico na manutenção da continência urinária e fecal tem salientado a necessidade de maiores informações quanto às suas propriedades contráteis. Os músculos do assoalho pélvico, quando normais, devem demonstrar tônus de relaxamento e a habilidade de contrair e relaxar tanto reflexamente como voluntariamente, e também de auxiliar na ação dos esfíncteres da uretra, vagina, e reto (GOSLING et al., 1981; BO; SHERBURN, 2005; MESSELINK et al., 2005).

Os músculos do assoalho pélvico compreendem o diafragma pélvico, o qual é uma divisão fibromuscular afunilada que forma a estrutura de sustentação primária do conteúdo pélvico (POLDEN; MANTLE, 1993; MORENO, 2004). O diafragma pélvico tem a forma de tenda invertida, e insere-se, lateralmente, num nível mais elevado do que a porção central, que é mais baixa (MORENO, 2004; BEREK, 2008). É composto pelos músculos levantador do ânus e coccígeos, juntamente com suas fáscias superiores e inferiores e forma o teto da fossa isquiorretal (MORENO, 2004).

O músculo elevador do ânus é adaptado para mantero tônus porperíodos prolongados, assim como para resistir a ascensões repentinas em pressão intraabdominal, como, por exemplo, ao tossir, espirrar ou correr. Isto se dá devido ao fato de possuir dois tipos de fibras musculares, tipo 1 (contração lenta) e tipo 2 (contração rápida) (POLDEN; MANTLE, 1993; MORENO, 2004).

As fibras do tipo 1 são altamente resistentes e, conseqüentemente, produzem a contração por longos períodos, apesar da força de contração tender a ser de ordem relativamente baixa. Já as fibras do tipo 2 são altamente exaustíveis, mas produzem alta ordem de força na contração rápida (MORENO, 2004).

A contração voluntária do assoalho pélvico age mais especificamente nas fibras musculares tipo 2, promovendo hipertrofia e potencializando a força de contração perineal (GROSSE; SENGLER, 2001; OLIVEIRA; LEMGRUBER, 2001; MORENO, 2004). Como os músculos levantadores do ânus desempenham papel importante no suporte pélvico e uretral, estudos sugerem que o exercício poderia melhorar a sustentação da uretra proximal, gerando aumento da continência durante a tosse (POLDEN; MANTLE, 1993; GROSSE; SENGLER, 2001; OLIVEIRA; LEMGRUBER, 2001; MORENO, 2004).

É de conhecimento que músculos sadios são volumosos e isso os capacita a suportar as paredes da vagina, e prover uma ação esfincteriana para a vagina e uretra. Com isso, eles favorem o fechamento e continência uretral (GROSSE; SENGLER, 2001; BEREK, 2008). É plausível que o suprimento de sangue associado a músculos fortes promoverá a saúde do epitélio na área, estimulando a lubrificação vaginal adequada, aumentando a resistência à infecção e retardando as mudanças atróficas da idade (POLDEN; MANTLE, 1993).

O períneo é a área em forma de losango medial às coxas e nádegas tanto dos homens quanto das mulheres que contém os órgãos genitais externos e o ânus (GUYTON; HALL, 2002; TORTORA; GRABOWSKI, 2002; MORENO, 2004). A região entre a vagina e o ânus corresponde ao centro tendíneo do períneo, local em que a maioria dos músculos perineais se insere. Esse local, também chamado de corpo perineal, determina um local de resistência (TORTORA; GRABOWSKI, 2002; MORENO, 2004).

Climatério é a fase da vida da mulher 
compreendida entre o final da vida reprodutiva e o início da senilidade, marcada por eventos importantes como a última menstruação e a menopausa (OLIVEIRA; LEMGRUBER, 2001; MOREIRA et al., 2002).

Nesse período, ocorrem as manifestações vasomotoras representadas pelas ondas de calor e sudorese, conseqüentes a queda da produção de estradiol pelos ovários. O climatério ocorre geralmente em mulheres com mais de 45 anos de idade, quando não mais existe a capacidade de se reproduzir naturalmente, em virtude do esgotamento dos folículos ovarianos, e da diminuição da produção hormonal pelos ovários (OLIVEIRA; LEMGRUBER, 2001; MOREIRA et al., 2002).

A principal função do estrogênio consiste em determinar a proliferação e o crescimento celular dos tecidos dos órgãos sexuais e de outros tecidos relacionados com a reprodução (FONSECA, 2000; GUYTON; HALL, 2002).

O climatério compreende, portanto, a fase pré-menopausa com duração variável de alguns anos, a partir do aparecimento de irregularidades menstruais; e um ano pós-menopausa (BEREK, 2008; FONSECA, 2000; GUYTON; HALL, 2002).

No climatério e, principalmente, no período pósmenopausa, ocorrem modificações fisiológicas em todo o organismo (FONSECA, 2000; GUYTON; HALL, 2002; BEREK, 2008). O assoalho pélvico sofre as conseqüências da progressiva atrofia músculo-aponeurótica e conjuntiva. Se houver lesões obstétricas associadas, surgem o prolapso e a incontinência urinária de esforço (FONSECA, 2000; PEREIRA; SOARES, 2001).

A idade também está associada com a diminuição do número de fibras de contração rápida; o confinamento ao leito ou o sedentarismo, a obesidade e a obstipação também leva à atrofia destas mesmas fibras (ROTHSTEIN, 1982). É relatado que a função do músculo estriado diminui cerca de $1 \%$ ao ano, após o pico na meia-idade, esta diminuição cumulativa associa-se à lesão do tecido conjuntivo (FONSECA, 2000; PEREIRA; SOARES, 2001; MARINHO; ARAÚJO, 2004; BARBOSA et al., 2005).

Cabral, Pereira e Aldrighi (2005) afirmam que há significativa taxa de sedentarismo, tornando imperiosa a criação de programas educativos e informativos no sentido de estimular a prática regular de atividade física, principalmente em mulheres no climatério (40 a 65 anos), quando são mais prevalentes os agravos que sabidamente melhoram após atividade física regular. A atuação da fisioterapia nesse estágio da vida feminina tem por finalidade promover a prática de exercícios específicos, para amenizar os efeitos das alterações fisiológicas e, principalmente, prevenir alterações musculoesqueléticas, proporcionando à mulher bem-estar e melhor qualidade de vida (MARINHO; ARAÚJO, 2004).

A avaliação da contração voluntária dos músculos do assoalho pélvico envolve a habilidade de elevar o assoalho pélvico tanto quanto a manutenção da força, endurance e coordenação muscular (BO; SHERBURN, 2005).

Moreira (1999), ao utilizar a avaliação da força muscular do assoalho pélvico por meio da palpação bidigital e perineometria, observou que mulheres incontinentes tinham déficit significativo da força muscular e da percepção do assoalho pélvico, comparadas às continentes.

A avaliação da musculatura do assoalho pélvico é de suma importância na eleição do tratamento apropriado para a incontinência urinária e/ou fecal, prolapsos de órgãos pélvicos e disfunção sexual. É através da manutenção da contração da musculatura perineal, ou seja, da força apresentada pela mesma, que se pode avaliar a sua funcionalidade na sustentação dos órgãos pélvicos e na geração e manutenção da pressão positiva uretral feminina (BO; SHERBURN, 2005).

A Fisioterapia tem como objetivos a prevenção, cura, reabilitação e reeducação de uma gama variada de patologias, dentre as quais se destaca 
a incontinência urinária pela sua alta incidência, cujo principal foco de atendimento é a reeducação períneo-esfincteriano. Porém não existe na literatura uma padronização de qual é a força muscular do assoalho pélvico normal para mulheres em determinadas faixas etária. Os estudos salientam apenas a diminuição da força muscular do assoalho pélvico e sua correlação com a incontinência urinária e/ou fecal, bem como os métodos de reabilitação e fortalecimento muscular do assoalho pélvico e a sua correlação com a continência urinária e/ou fecal.

O objetivo deste estudo foi comparar a força de pressão muscular do assoalho pélvico de mulheres jovens, entre 25 e 35 anos, com a de mulheres climatéricas entre 45 e 55 anos, buscando identificar se a força muscular do assoalho pélvico diminui com o avançar da idade.

\section{Material e métodos}

O delineamento foi um estudo comparativo transversal. A população foi constituída de alunas de uma instituição de ensino superior e funcionárias do Hospital Universitário, nas faixas etárias entre 25 a 35 e 45 a 55 anos, correspondentes, respectivamente, à idade da menacme e do climatério. A população composta por 49 mulheres saudáveis, divididas em dois grupos; Grupo 1, composto por 24 mulheres com média de idade de 29,33 $( \pm 2,86)$ anos e o Grupo 2, composto por 25 mulheres com média de idade de 51,8 $( \pm 4,5)$ anos (TABELA 1). Foram excluídas do estudo, mulheres virgens, portadoras de doença neurológica e/ou desmielinizante, com presença de prolapso vaginal anterior e/ou posterior de grau II ou III, com presença de infecção vaginal, com sintomas de incontinência urinária e/ou fecal, mulheres submetidas a cirurgias uroginecológicas prévias e grávidas.

As participantes foram esclarecidas sobre os procedimentos a ser realizado e sobre os objetivos deste estudo, sendo necessária assinatura do "Termo de Consentimento livre e Esclarecido", aprovado pelo Comitê de Pesquisa e Ética do Hospital
Universitário/UEL, parecer número 306/04.

As mulheres foram avaliadas no ambulatório de fisioterapia do Hospital Universitário/UEL (Londrina-PR), por meio de avaliação clínica fisioterápica da musculatura do assoalho pélvico que consistia de: palpação bidigital, onde a mulher era solicitada que contraísse o assoalho pélvico contra os dedos do avaliador e sustentasse a contração por mais de 5 segundos. A contração era classificada como: ausente; presente e não sustentada por mais de 5 segundos; presente e sustentada por mais de 5 segundos.

A força de pressão muscular do assoalho pélvico foi avaliada por meio de um perineômetro (Peritron 9300+), constituído de sensor vaginal que possibilita a medida do pico máximo de contração (fibras de contração rápida, tipo II) $\left(\mathrm{em}^{\mathrm{cmH}} \mathrm{H}_{2} \mathrm{O}\right)$, a média de sustentação (fibras de contração lenta, tipo I) $\left(\right.$ em $\left.\mathrm{cmH}_{2} \mathrm{O}\right)$ e o tempo de duração da contração muscular (endurance) (em segundos).

Durante toda a avaliação clínica, foi observado e anotado o uso de musculatura acessória.

A avaliação clínica foi realizada com a paciente em consultório individual, na posição de litotomia, em mesa ginecológica, utilizando luvas de procedimento e preservativo.

Para a análise estatística utilizou-se a média, desvio padrão e o Teste $\mathrm{t}$ de Student para amostras independentes e para comparação entre os grupos, utilizando o software SPSS 11.5 para Windows. Todos os testes estatísticos foram realizados com significância de $5 \%(p<0,05)$.

\section{Resultados e discussão}

O número de pacientes mais velhas que buscam cuidado para redução de sintomas urinários continuará aumentando durante as próximas décadas, devido ao aumento na expectativa e qualidade de vida das mulheres (MARINHO; ARAÚJO, 2004). Estudos das mudanças anatômicas e fisiológicas relacionadas ao envelhecimento 
nestas estruturas é fonte de pesquisas e estão sendo crescentemente compreendidas, e isso tem auxiliado a prevenção de sintomas do trato urinário inferior como os prolapsos e a incontinência urinária e fecal (OUSLANDER, 1997; MARINHO; ARAÚJO, 2004; BARBOSA et al., 2005; TROWBRIDGE et al., 2007).

$\mathrm{Na}$ avaliação da história obstétrica notou-se diferença estatística entre os grupos no número de gestações e partos vaginais o que não ocorreu com relação ao número de cesáreas (Tabela 1).

Tabela 1 - Caracterização dos grupos estudados, média, desvio padrão e resultado estatístico, Londrina-PR, 2008.

\begin{tabular}{cccc}
\hline VARIÁVEIS & GRUPO 1 $(N=24)$ & GRUPO 2 $(N=25)$ & Resultado estatístico \\
\hline IDADE & $29,33 \pm 2,86$ & $51,8 \pm 4,5$ & $\mathrm{p}=0,000^{*}$ \\
GESTAÇÃO & $1,08 \pm 1,14$ & $3,16 \pm 1,75$ & $\mathrm{p}=0,000^{*}$ \\
PARTOS & $0,29 \pm 0,55$ & $2,04 \pm 1,9$ & $\mathrm{p}=0,000^{*}$ \\
CESÁREAS & $0,67 \pm 0,92$ & $1,04 \pm 1,1$ & $\mathrm{p}=0,20$ \\
\hline
\end{tabular}

*significativo ao nível de $95 \%(\mathrm{p}<0,05)$

Neste estudo observou-se que, na avaliação pela palpação bidigital da musculatura do assoalho pélvico, 95,8\% das mulheres do Grupo 1 e 76\% das mulheres do grupo 2 foram capazes de contrair a musculatura do assoalho pélvico contra os dedos do avaliador e manter a contração por mais de 5 segundos (FIGURA 1). Este achado indica maior capacidade de contração voluntária e sustentação muscular nas mulheres com faixa etária entre 25 a 35 anos. O mesmo resultado foi encontrado em estudo realizado na UFP/ Pará, no qual se comparou a força da musculatura perineal em mulheres com e sem incontinência urinária de esforço, no menacme e na menopausa. Utilizando cones vaginais, evidenciou-se maior força muscular nas mulheres continentes, quando comparadas às incontinentes (respectivamente no menacme e na pós-menopausa) (MOREIRA et al., 2002).

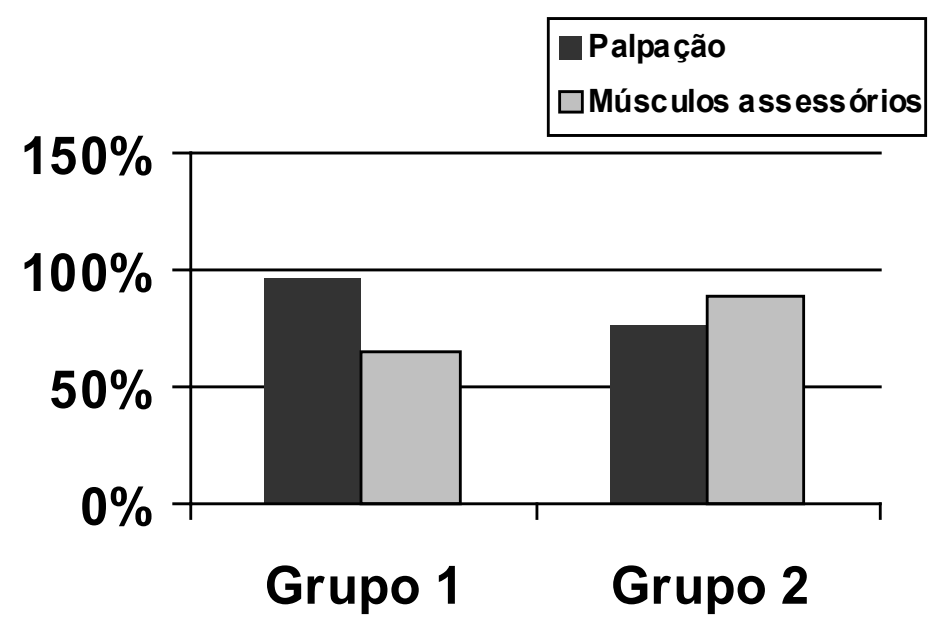

Figura 1 - Porcentagem da avaliação pela palpação bidigital e utilização de músculos assessórios entre os grupos estudados, Londrina-PR, 2008. 
$\mathrm{Na}$ avaliação da força de pressão muscular do assoalho pélvico, verificou-se que o pico da contração muscular do assoalho pélvico, ou seja, a força de pressão das fibras musculares do tipo II, foi de 42,21 $( \pm 20,7) \mathrm{cmH}_{2} \mathrm{O}$ no grupo 1 contra $36,92( \pm 15,01)$ no grupo $2(p>0,05)$. Apesar de não haver diferenças significantes, os valores da média de contração nos indicam melhor desempenho nas mulheres do grupo 1 (Figura 2).

Resultados semelhantes foram obtidos na média de sustentação da contração muscular, fibras do tipo I, onde as mulheres do grupo 1 apresentaram média de $30,3( \pm 17,9) \mathrm{cmH}_{2} \mathrm{O}$ e nas do grupo 2 foi de $25,8( \pm 11,3) \mathrm{cmH}_{2} \mathrm{O}(\mathrm{p}>0,05)$ (Figura 2).

Com relação à duração da contração muscular, as mulheres de ambos os grupos não apresentaram diferenças significantes, obtendo o grupo 1 média de 13,5 $( \pm 4,1)$ segundos e o grupo 2 média de 14,5 $( \pm 3,5)$ segundos, indicando endurance muscular semelhante $\mathrm{p}>0,05$ (Figura 2).

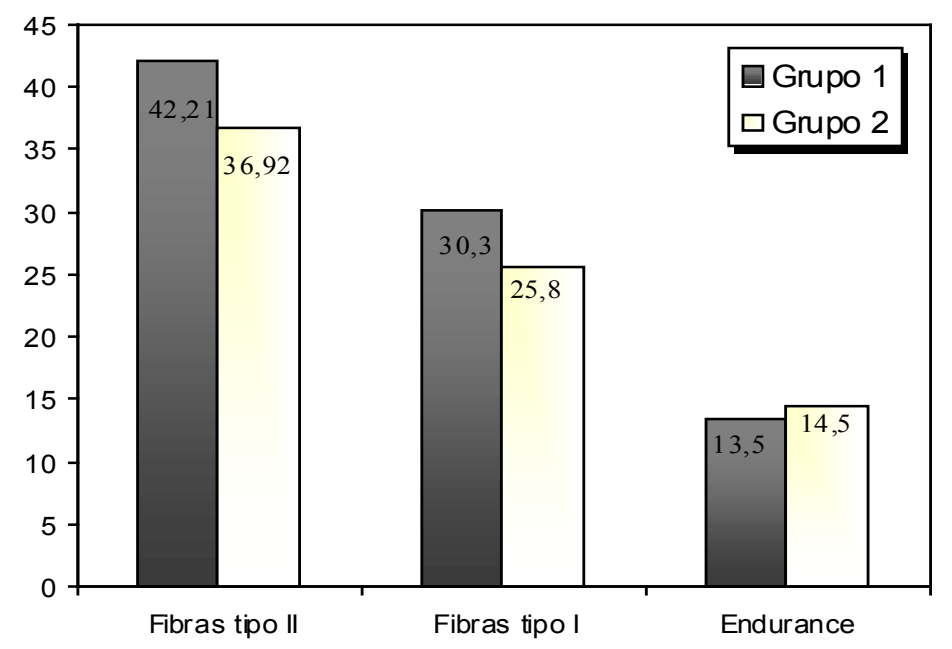

Figura 2 - Média da força de pressão muscular das fibras tipo II $\left(\mathrm{cmH}_{2} \mathrm{O}\right)$, fibras tipo I $\left(\mathrm{cmH}_{2} \mathrm{O}\right)$ e da endurance muscular (segundos) nos grupos estudados, Londrina-PR, 2008.

Em um estudo com o objetivo de avaliar os efeitos do envelhecimento, no suporte de apoio pélvico e uretral, função uretral e do assoalho pélvico, foram avaliadas 82 mulheres nuliparas entre 21 e 70 anos. Os autores notaram resultados semelhantes a este estudo onde não houve diferenças clínicas nas medidas de suporte pélvico e nelas se inclui os músculos que fazem parte do assoalho pélvico nas diferentes idades. No entanto, observou-se que a pressão máxima de fechamento uretral foi $40 \%$ melhor nas mulheres jovens (TROWBRIDGE et al., 2007).

DeLancey, Gosling e Creed (2002) realizaram estudo histológico em mulheres nulíparas assintomáticas, identificando evidências de alterações do assoalho pélvico como a denervação e alterações do tipo, número e diâmetro das fibras musculares.

Norton (1993) observou que, nas mulheres mais velhas, ocorre a redução de colágeno nos músculos pélvicos e na fáscia, associado com o aumento de pontes cruzadas de tecido fibroso o que diminui a elasticidade, e, portanto a força muscular.

Estudos comparativos entre a força de pressão muscular do assoalho pélvico entre mulheres jovens e climatéricas são em número reduzido, porém os 
estudos com perineometria de mulheres climatéricas demonstraram valores semelhantes aos encontrados neste estudo (KERSCHAN-SCHINDL et al., 2002; HUNDLEY; WU; VISCO, 2005).

O presente estudo também identificou que $65 \%$ das mulheres do grupo 1 e $89 \%$ das mulheres do grupo 2 fizeram uso de musculatura assessória durante a avaliação (FIGURA 1), sendo os grupos musculares mais utilizados, os glúteos, os abdominais, os adutores, e também a manobra de valsalva. A utilização dessa musculatura não interferiu nos resultados, pois o sítio de captação da pressão muscular do perineômetro está localizado nos $3 \mathrm{~cm}$ superiores da sonda endovaginal. Porém vale lembrar que estudos identificaram a presença de sinergia abdômino-pélvica durante a execução de manobras respiratórias, bem como acréscimo na pressão perineal e no sinal eletromiográfico perineal e abdominal durante a execução das manobras respiratórias (SAPSFORD et al., 2001; NAGIB et al., 2005). Isso nos remete à hipótese da influência deste sinergismo nos valores da contração perineal durante a realização do teste.

Alguns estudos relatam que muitas mulheres apresentam dificuldades em realizar uma contração correta dos MAP na primeira avaliação, e isso sugere que a elevação do assoalho pélvico não é uma resposta automática à solicitação de "contrair e sustentar" o assoalho pélvico (THOMPSON; O'SULLIVAN, 2003; TALASZ et al., 2005; THOMPSON et al., 2006b).

Thompson et al. (2006a) observaram que, durante a avaliação da contração do assoalho pélvico ocorre manobra de valsalva em $25 \%$ a $43 \%$ das mulheres com incontinência urinária e prolapso. Em outros estudos Thompson et al. (2006b) notaram que $17 \%$ das mulheres continentes também realizavam valsalva durante a avaliação, o que reforça a necessidade de orientações e da especificidade durante o treino da contração dos músculos do assoalho pélvico. Os mesmos autores afirmam que mulheres continentes apresentam maior força e endurance dos MAP quando comparadas a mulheres incontinentes.

\section{Conclusões}

O presente estudo não identificou diferenças significativas da força de pressão muscular do assoalho pélvico pela perineometria entre os grupos estudados.

$\mathrm{Na}$ avaliação pela palpação bidigital, notou-se uma capacidade maior de contração voluntária e sustentação desta contração nas mulheres com faixa etária entre 25 a 35 anos.

As mulheres do grupo 2 utilizaram mais os músculos assessórios durante os procedimentos de avaliação.

Acredita-se que mais estudos, com uma amostra maior, são necessários para a afirmação deste achado.

\section{Referências}

BARBOSA, A. M. P.; CARVALHO, L. R.; MARTINS, A. M. V. C.; CALDERON, I. M. P.; RUDGE, M. V. C. Efeito da via de parto sobre a força muscular do assoalho pélvico. Revista Brasileira de Ginecologia e Obstetrícia, São Paulo, v. 27, n. 11, p. 677-682, 2005.

BEREK J. S. Novak: tratado de ginecologia. 14. ed. Rio de Janeiro: Guanabara Koogan, 2008.

BO, K.; SHERBURN, M. Evaluation of female pelvicfloor muscle function and strength. Physical Therapy, Alexandria, v. 85, n. 3, p. 269-282, 2005.

CABRAL, A. F. G. C. M.; PEREIRA, E. C. A.; ALDRIGHI, J. M. Quais os benefícios da atividade física no climatério? Revista da Associação Médica Brasileira, São Paulo, v. 51, n. 4, p. 183-183, 2005.

DELANCEY, J.; GOSLING, J.; CREED, K. Gross anatomy and cell biology of the lower urinary tract. In: ABRAMS, P.; CARDOZO, L.; KHOURY, S. (Ed.). Incontinence: 2nd international consultation on incontinence. Plymouth: Health Publication, 2002. p. $19-82$.

FONSECA, A. M. Fisiologia do climatério. In: HALBE, H. W. Tratado de ginecologia. 3. ed. São Paulo: Roca, 2000. v. 2, p. 1237-1242. 
GOSLING, J. A.; DIXON, J. S.; CRITCHLEY, H. O. D.; THOMPSON, S. A. A comparative study of the human external sphincter and periurethral levator ani muscles. British Journal of Urology, Oxford, v. 53, n. 1, p. 35-41, 1981.

GROSSE, D.; SENGLER, J. Reeducação perineal. São Paulo: Manole, 2001.

GUYTON, A. C.; HALL, J. E. Tratado de fisiologia médica. 10. ed. Rio de Janeiro: Guanabara Koogan, 2002. p. 59-82.

HUNDLEY, A. F.; WU, J. M.; VISCO, A. G. A comparison of perineometer to brink score for assessment of pelvic floor muscle strength. American Journal of Obstetrics \& Gynecology, New York, v. 192, n. 5, p. 1583-1591, 2005 .

KERSCHAN-SCHINDL, K.; UHER, E.; WIESINGER, G.; KAIDER, A.; EBENBICHLER, G.; NICOLAKIS, P.; KOLLMITZER, J.; PREISINGER, E.; FIALKAMOSER, V. Reliability of pelvic floor muscle strength measurement in elderly incontinent women. Neurourol and Urodynamics, New York, v. 21, n. 1, p. 42-47, 2002.

MARINHO, A. C. N.; ARAÚJO, T. Fisioterapia e climatério. Fisioterapia Brasil, Rio de Janeiro, v. 5, n. 5, p. 405-409, 2004.

MESSELINK, B.; BENSON, T.; BERGHMANS, BO, K.; CORCOS, J.; FOWLER, C.; LAYCOCK, J.; LIM, P. H.; VAN-LAUSEN, R.; NIJEHOLT, G. L.; PEMBERTON, J.; WANG, A.; WATIER, A.; VAN-KERREBROECK, P. Standardisation of terminology of pelvic floor muscle function and dysfunction: report fromthe pelvic floor clinical assessment group of the international continence society. Neurourol and Urodynamics, New York, v. 24, n. 4, p. 374-380, 2005.

MOREIRA, E. C. H. Valor da avaliação objetiva e subjetiva no diagnóstico da incontinência urinária feminina: correlação com a força muscular do assoalho pélvico. 1999. 124 fls. Dissertação (Mestrado em Ginecologia e Obstetrícia) - Universidade Estadual Paulista, Faculdade de Medicina, Botucatu.

MOREIRA, S. F. S.; GIRÃO, M. J. B. C.; SATORI, M. G. F.; BARACAT, E. C.; LIMA, G. R. Mobilidade do colo vesical e avaliação funcional do assoalho pélvico em mulheres continentes e com incontinência urinária de esforço, consoante o estado hormonal. Revista Brasileira de Ginecologia e Obstetrícia, São Paulo, v. 24, n. 6, p. 365-370, 2002.

MORENO, A. L. Fisioterapia em uroginecologia. São Paulo: Manole, 2004.
NAGIB, A. B. L.; GUIRRO, E. C. O.; PALAURO, V. A.; GUIRRO, R. R. J. Avaliação da sinergia da musculatura abdomino-pélvica em nulíparas com eletromiografia e biofeedback perineal. Revista Brasileira de Ginecologia e Obstetetrícia, São Paulo, v. 27, n. 4, p. 210-215, 2005.

NORTON, P. A. Pelvic floor disorders: the role of fascia and ligaments. Clinical Obstetrics and Gynecology, Hagerstown, v. 36, n. 4, p. 926-938, 1993.

OLIVEIRA, H. C.; LEMGRUBER, I. Tratado de ginecologia febrasgo. Rio de Janeiro: Revinter, 2001.

OUSLANDER, J. G. Aging and the lower urinary tract. The American Journal of Medical Sciences, Philadelphia, v. 314, n. 4, p. 214-218, 1997.

PEREIRA, A. S. F.; SOARES, A. Endocrinologia da perimenopausa. In: OLIVEIRA, H. C.; LEMGRUBER, I. Tratado de ginecologia febrasgo. Rio de Janeiro: Revinter, 2001. p. 653-654.

POLDEN, M.; MANTLE, J. Fisioterapia em ginecologia e obstetrícia. São Paulo: Santos, 1993.

ROTHSTEIN, J. M. Muscle biology: clinical considerations. Physical Therapy, Alexandria, v. 62, n. 1, p. 1823-1830, 1982.

SAPSFORD, R. R.; HODGES, P. W.; RICHARDSON, C. A.; COOPER, D. H.; MARKWELL, S. J.; JULL, G. A. Co-activation of the abdominal and pelvic floor muscles during voluntary exercises. Neurourology and Urodynamics, New York, v. 20, n. 1, p. 31-42, 2001.

TALASZ, H.; GOSCH, M.; ENZELSBERGER, H.; RHOMBERG, H. P. Geriatrische patientinnen mit harninkontinenz-symptomen und ihre kontrolle über den beckenboden. Zeitschrift für Gerontologie und Geriatrie, Darmstadt, v. 38, n. 6, p. 424-430, 2005.

THOMPSON, J. A.; O'SULLIVAN, P. B. Levator plate movement during voluntary pelvic floor muscle contraction in subjects with incontinence and prolapse: a cross sectional study and review. International Urogynecology Journal and Pelvic Floor Dysfunction, Surrey, v. 12, n. 4, p. 84-88, 2003.

THOMPSON, J. A.; O'SULLIVAN, P. B.; BRIFFA, N. K.; NEUMANN, P. Altered muscle activation patterns in symptomatic women during pelvic floor muscle contraction and valsalva manouevre. Neurourology and Urodynamics, New York, v. 25, n. 3, p. 268-276, 2006 a.

Assessment of voluntary pelvic floor muscle contraction in continent and incontinent women using transperineal ultrasound, manual muscle testing and vaginal pressure measurements. International Urogynecology Journal and Pelvic Floor Dysfunction, Surrey, v. 12, n. 3, p. 166-169, 2006b. 
TORTORA, G.; GRABOWSKI, S. R. Princípios de anatomia e fisiologia. 9. ed. Rio de Janeiro: Guanabara Koogan, 2002.
TROWBRIDGE, E. R.; WEI, J. T.; FENNER, D. E.; ASHTON-MILLER, J. A.; DELANCEY, J. O. L. Effects of aging on lower urinary tract and pelvic floor function in nulliparous women. Obstetrics \& Gynecology, New York, v. 109, n. 3, p. 715-720, 2007.

Recebido em 5 de março de 2008 - Received on March 5, 2008. Aceito em 13 de maio de 2009 - Accepted on May 13, 2009. 